UCRL-JC-130404

PREPRINT

Mirror-Based Fusion: Some Possible New Directions

\author{
R. F. Post
}

This paper was prepared for submittal to the

International Conference on Open Magnetic Systems for Plasma Confinement Novosibirsk, Russia

July 27-31, 1998

July 16, 1998

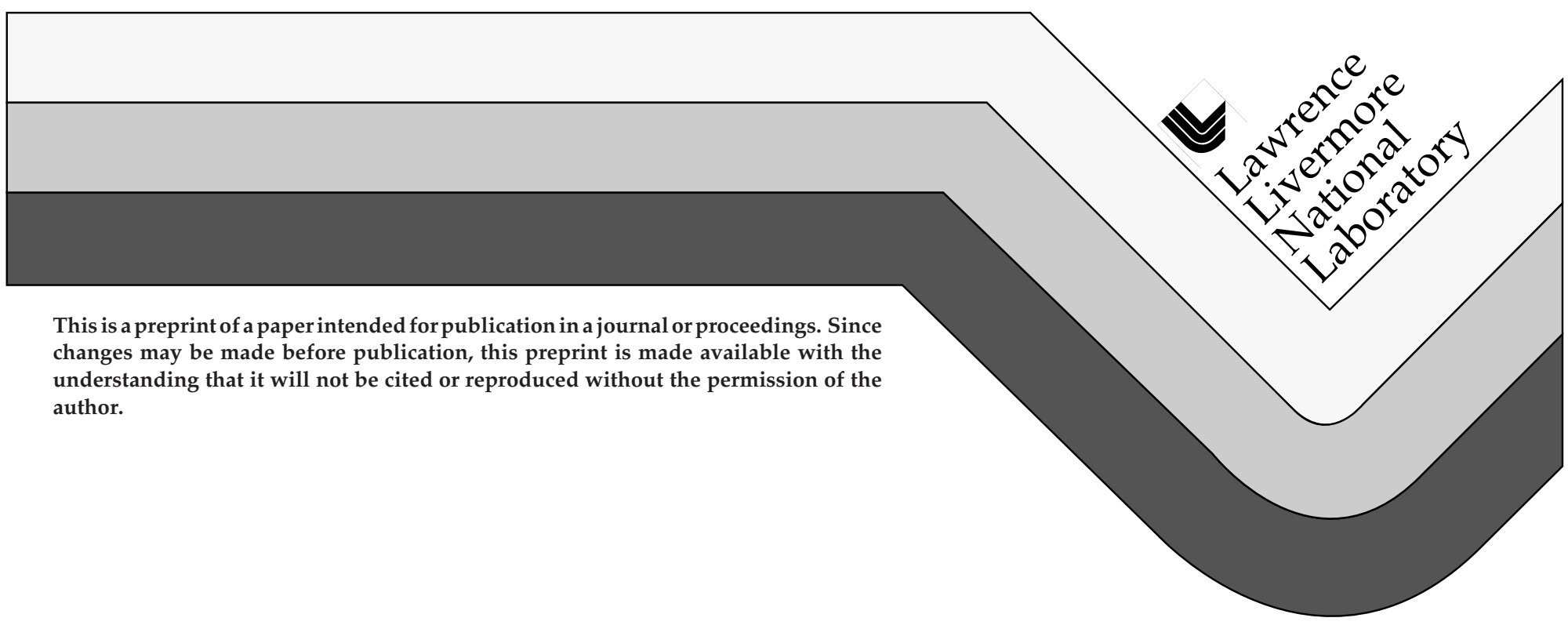




\section{DISCLAIMER}

This document was prepared as an account of work sponsored by an agency of the United States Government. Neither the United States Government nor the University of California nor any of their employees, makes any warranty, express or implied, or assumes any legal liability or responsibility for the accuracy, completeness, or usefulness of any information, apparatus, product, or process disclosed, or represents that its use would not infringe privately owned rights. Reference herein to any specific commercial product, process, or service by trade name, trademark, manufacturer, or otherwise, does not necessarily constitute or imply its endorsement, recommendation, or favoring by the United States Government or the University of California. The views and opinions of authors expressed herein do not necessarily state or reflect those of the United States Government or the University of California, and shall not be used for advertising or product endorsement purposes. 


\title{
MIRROR-BASED FUSION: SOME POSSIBLE NEW DIRECTIONS
}

\author{
Richard F. Post \\ Lawrence Livermore National Laboratory \\ Mail Stop L-644 P.O. Box 808 \\ Livermore, California 94550
}

(925) 422-9853

\begin{abstract}
This paper examines some possible areas for the study of new approaches to fusion research, ones that employ magnetic confinement systems based on openended field topology and employing the magnetic mirror principle. In the spirit of encouraging a wider look at possibilities, some unconventional approaches are suggested. These approaches, involving long linear systems having ion injectors and direct converters at their ends, attempt to exploit some inherent advantages of open-ended systems for fusion. The results of analysis, calculations and preliminary cost estimates for long linear systems of this type that utilize the magnetic mirror effect to achieve their operating regimes will be presented. The approaches suggested, when examined in greater depth, may not stand the test of time, but they might encourage thinking in new areas.
\end{abstract}

\section{INTRODUCTION}

The scientific quest for fusion power has now been pursued for half a century. As a result of this quest major advances have been made in the physics of hightemperature plasmas. Despite these advances no wholly satisfactory approach to fusion has as yet been found. We are therefore presented with both an opportunity and a challenge. The opportunity: better approaches to fusion are both needed and wanted. The challenge: to rethink present images of fusion power systems in order to uncover new approaches. However, unlike innovation in the early days of fusion research, which was not grounded in a thorough understanding of the plasma state, today we can call on a detailed knowledge of that state in searching for better approaches. Another aid for such a search is that fusion, among all potential sources of energy, has the largest volume of parameter space - fuel resources, fuel densities, and power densities within which to pursue such a search. Thus, though the most difficult to achieve of all energy sources, fusion presents the largest number of avenues to follow in looking for new approaches.

In contemplating new approaches two decisions must be made. One of these is the plasma regime to be considered. By "regime" is meant not only the temperature and the density of the plasma, but also the degree of order of the particles. The conventional degree of order is a near-maxwellian state, with particle containment lifetimes being much longer than collision times, and with the plasma being well characterized by the MHD fluid model. At the other extreme are beamlike plasmas, with ions and/or electrons far from collisional equilibrium. However, being simple binary reactions, fusion reactions are indifferent as to whether they occur between ions in the former or the latter state. The examples given are in the latter state, so that the particle-orbit picture of plasma is a better model than the MHD-fluid model.

The second decision to be made in looking for new magnetic confinement systems is the topology of the magnetic field: "open" or "closed." It has long been the author's contention that the open topology is more conducive to innovation and more flexible in operating regimes and technology than closed systems [1]. We therefore chose open systems for the examples, specifically, long linear systems located in underground tunnels, following up on earlier calculations [2]. At the ends of these systems are located ion sources and electrostatic direct converters. The ion sources are used to create the plasma regimes at the kinetic energies needed for fusion, and the direct converters are used recover energy, at high efficiency, both from fusion 
reaction products and from unreacted fuel ions. Thus a state of plasma ignition is not required to achieve a net positive energy release.

In locating these systems in underground tunnels we borrow a leaf from the book of high-energy physicists, who in this way have found a solution to the land-use problem posed by many-kilometer-long facilities. An example of such a facility is the 27 kilometer-long Large Electron-Proton Collider at CERN in Switzerland. The tunnel for this accelerator runs below farmland and villages in both Switzerland and France with no interference with those land uses above the tunnel.

\section{SOME ECONOMIC CONSIDERATIONS}

For our proposed systems, at any reasonable fusion power level per meter of the fusion system the cost of tunneling will be small compared to the revenue per meter obtained from the generated fusion power over the lifetime of the facility. This being the case the economic feasibility of long linear fusion systems in underground tunnels will depend almost entirely on the capital cost per meter of such systems.

Some preliminary economic considerations for long linear fusion systems were given in a previous report [3]. The long linear systems that will be described have as their main elements modular solenoidal coils. An approximate estimate of the cost of such coils can be made by referring to cost studies for large SMES (Superconducting Magnetic Energy Storage) systems [5]. Converting their cost in Joules $/ \mathrm{m}^{3}$ to costs of highfield solenoidal magnets one finds a cost of about $\$ 30,000$ per meter of magnet coil. As a rough estimate of the cost of our linear fusion system we will multiply this cost by a factor of five, to obtain a cost of $\$ 150,000$ per meter. Assume now that $\$ .02 / \mathrm{kWh}$ from the revenue of the plant can be set aside to amortize its construction over an assumed 20 year lifetime. At an assumed operating factor of 85 percent for the plant the electrical power output of the plant must be about 50 kilowatts/meter to meet this requirement. Assuming an overall conversion efficiency of the plant of 33 percent, generating this electrical power output per meter requires $150 \mathrm{~kW} / \mathrm{m}$ of fusion power. Yields of this level or above would satisfy the economic pay-back requirement as defined above.

The above economic estimates are admittedly approximate. They do show, however, that if power outputs per meter of the order of those listed can be achieved, an economic case for long linear systems can be made. In the examples to be given fusion power densities are projected to be in excess of 10 megawatts/cubic meter (for D-T ). To yield $150 \mathrm{~kW} / \mathrm{m}$ of fusion power from a linear system at this fusion power density would then imply a plasma column radius of about .07 meters, small as compared to plasma radii in typical tokamak systems.

\section{OPEN-SYSTEMS: PHYSICS CONSIDERATIONS}

We will be considering open-ended fusion systems employing the magnetic-mirror effect. There are several plasma-physics attributes associated with such systems. We review these here as a basis for later discussions. Recapitulating from earlier reports [6], these attributes are:

1. Open-ended topology permits confinement in regions where the field-line curvature is everywhere positive, thereby suppressing interchange-type MHD instability modes. This property has been shown to allow the achievement of MHD-stable plasmas with plasma beta values approaching unity [7], in agreement with theory [8].

2. Owing to the loss channel at the ends of the system, open-ended systems can confine a plasma column whose radial boundary need not terminate on a physical surface, but can exist far from the vacuum chamber wall if necessary. In the case in toroidal systems (which have only a single loss channel - i.e., radially, across the field lines) the plasma density and temperature must fall to zero at its boundary, thereby introducing difficult problems of local turbulence and plasma-wall interactions.

3. Open field-line topology permits the introduction of particle beams or plasma entities along the field lines into the confinement region. This property, one that was first exploited in the multistage magnetic compression mirror experiment at the Laboratory [9], could have important uses in the fueling and ash removal of the fusion plasma.

4. Just as open-field geometry permits the introduction of particles through the ends, it permits a high degree of control over their loss. After exiting the ends the particles can be collected on large-area surfaces that are located far from the confinement region, greatly reducing the problem of wall bombardment. It also opens up the possibility of using electrostatic direct converters [10] at the ends to recover kinetic energy, at high efficiency [11], from escaping particles, namely, from charged fusion reaction products and unreacted plasma ions (and electrons). 
5. By exploiting the special properties of the magnetic mirror effect it is possible to establish and control electric potentials within the plasma $[12,13]$. These potentials can be used to enhance the confinement, as in tandem mirror systems [14,15], or to control the direction of exit of plasma ions. The potential differences established can be either localized, as in tandem-mirror systems, and/or can be established in the radial direction, relative to the containment chamber, as an aid to the suppression of certain instability modes [16].

6. As compared to closed, i.e., toroidal systems, where the plasma state is usually a collision-dominated one, open-ended systems can much more readily operate near to "collisionless" state, i.e., one where randomization of the ion distribution functions is not complete so that the ions (or electrons) can exist far from a maxwellian state. This capability could be important in cases where it may be important (as in the $\mathrm{D}-\mathrm{He} 3$ reaction) to minimize the number of satellite neutron-producing reactions. Such less-randomized distribution functions are typically more amenable to high-efficiency direct conversion than are maxwellian ion distributions.

In addition to the specific technical characteristic just listed, we list again a qualitative, non-quantifiable, attribute of open-ended systems, one that is especially important at this juncture in fusion research. This attribute is their flexibility. By "flexibility" is implied both the adaptability of open-ended systems to a wide variety of plasma regimes (high beta, high plasma temperature, non-maxwellian ion distributions, etc.) and their suitability for innovative improvements. An example of "innovative improvements" taken from the research history of open-ended systems is the introduction of electrostatic direct converters and radial electric field control in tandem mirror systems [17]. By contrast, in closed systems such as the tokamak, the requirement for field closure within the confinement chamber introduces some severe constraints, not only on the plasma beta values, and on the minimum plasma radius, but equally importantly, also on the ability to make innovative improvements to the system.

There is yet another important opportunity presented by the adaptability of open-ended systems to the use of high-efficiency direct converters. This opportunity is to remove the necessity for achieving fusion "ignition" in order to achieve useful net power from a fusion power plant. The oft-stated goal of tokamak research is to achieve an ignited state of the fusion fuel in which plasma temperatures, densities, and confinement times are sufficient to allow the fusion reactions to be selfsustaining. Such a state is commonly denoted by one in which the reaction "Q" factor (ratio of fusion energy release deposited in the plasma to heating power) approaches infinity. However, from the time when fusion research was first initiated, its implicit goal has been a pragmatic one: "to generate useful power at a competitive cost, and with superior environmental and safety characteristics as compared to alternative energy sources." In the search for better ways to solve the fusion problem it is the author's belief that one should be guided less by the requirement of plasma ignition, and more by remembering the pragmatic goal. Achieving high $Q$ values may turn out to be of secondary importance as compared to other criteria that are more significant in terms of practicality and economics.

There are in fact some major advantages in exploring approaches that do not require plasma ignition to yield net power. These approaches are the so-called "driven" systems, ones in which electrical power is recirculated in order to heat the fuel ions, but where the efficiency of heating and of energy recovery are high enough to yield a useful net energy release. In such systems the fusion $Q$ value may actually be less than unity for a system with net electrical power output. An obvious advantage of low-Q systems is the relaxation of plasma confinement time requirements that is implied. In terms of its effect on the conduct of fusion research, this relaxation of requirements opens up for study and exploitation concepts that may have been earlier rejected as being incapable of reaching ignition. It also would have the important effect of shifting the research burden from a quest for ever-better confinement to a more balanced one. That is, some of the burden can be shifted from confinement physics issues to the exploration of well-defined technological issues, such as the development of efficient ion injectors and direct converters.

There is another reason why undertaking the investigation of low-Q fusion systems that employ highefficiency direct converters may be particularly valuable. While the examples presented in this paper are systems that employ the D-T reaction, there are many reasons why it would be highly desirable to employ other fusion reactions, such as the D-He3 reaction, or possibly the $\mathrm{p}-\mathrm{B} 11$ reaction in future fusion power systems. These fusion fuels, having their energy release in charged particles, can take maximal advantage of direct conversion techniques. At the same time, in low-Q operation parasitic neutron-producing reactions can be minimized or eliminated completely. If we are therefore to look for new and better approaches 
to magnetic fusion, ones that minimize the problems of tritium handling, induced radioactivity, and radiationdamage associated with the neutron-producing D-T fuel cycle, we would be well advised to investigate approaches that extrapolate naturally to fuel cycles other than D-T.

Finally, what emerges from the considerations that have been listed above is a need for a major shift in emphasis in the plasma physics issues that would need to be addressed in pursuing the proposed new approaches to open-ended systems. While not always the case in closed systems such as the tokamak, the fluid-like or MHD model of the plasma is the one that is most naturally considered in conceptualizing their behavior. However, as noted earlier, in the kind of plasma research that the author feels will be needed to exploit these new areas, "beam-like" plasma regimes represent a better model than the fluid model. Fortunately, there is a substantial body of knowledge of such plasma regimes in place as a result of earlier research in mirror-based systems. Not only is this data base extensive, but also there exists a parallel body of knowledge from the particle accelerator community. Theory, computer codes, and experimental data from both the fusion research community and the accelerator community could be brought to bear to address these problems.

The change in viewpoint that is here called for is to place less emphasis on the fluid-like or statistical mechanics aspects of plasma behavior, and instead to shift the emphasis to a more deterministic view of the behavior of the ions of the plasma as these ions move in the combined magnetic and electrical fields to which they are subjected.

\section{MIRROR-BASED LONG LINEAR SYSTEMS}

The conventional view of mirror-based open-ended systems is that of a long solenoidal magnetic field terminated at its ends by regions of higher magnetic field - the mirrors. In the conventional tandem mirror $[14,15]$ there are located short containment regions - the "plugs," bounded at each end by additional mirrors. In all of these systems the magnetic mirrors are used either to provide axial confinement of the plasma ions directly, through mirror reflection, or indirectly, to create a localized axially confining potential, as in the tandem mirror.

By contrast in the approaches that are to be further explored here the magnetic mirror effect will not be used to confine the ions, but will instead be employed to "compress" plasma ions following their injection into the field. In both of the examples given the field configuration will be that of a long axially symmetric solenoidal field in which the field is maximal in the central region, tapering down to a low value near the ends. As described in previous papers [2,3], ions are to be injected near the ends of the system, where the magnetic field is much weaker than it is in the central region. In such a field the field-line curvature is everywhere positive, thus suppressing MHD instability modes of the interchange variety. Furthermore, since the field is axially symmetric there will not occur the type of resonant cross-field transport that can cause difficulties in non-axially symmetric mirror fields [18].

In these systems energetic ions are to be injected from ion or (neutral beam) sources having a sharply peaked angular distribution. Their angle of injection will be nearly parallel to the local direction of the magnetic field so that the effect of the magnetic field will be to guide the ions along the converging field lines, compressing their density and gradually increasing their pitch angle. Depending on the mode of operation desired the injected ions will either penetrate the field up to its maximum and subsequently escape out the far end (the "linear collider" mode), or will be stopped and reflected before reaching the maximum point in the field (the "kinetic tandem" mode [3]). In either case the exiting ions will be "decompressed" as they move down the field lines, have their perpendicular component of energy transformed into parallel motional energy as a result, and finally be collected by electrostatic direct converters that recover much of the energy invested in launching those ions.

In either of the systems to be discussed the achievement of fusion-relevant plasma conditions will be heavily dependent on technology-related factors such as the current density and angular distribution of the ion sources, the strength of the confining magnetic field, and the effectiveness of the direct converter systems. On the other hand, confinement-related issues such as MHD instabilities, turbulence-driven cross-field transport, and plasma-wall interactions will be expected to be far less important than they are in conventional fusion systems, such as the tokamak. Correspondingly, much of the research emphasis would be expected to shift away from such concerns to the problem of meeting some well-defined technology requirements. Though not trivial, the remaining confinement-related plasma physics issues should be highly amenable to analysis, computer simulation, and experimental test, undergirded by an already existent extensive data base from previous work on open-ended systems. 


\section{A. A Linear Collider}

From a confinement viewpoint, the simplest possible open-ended system would be the linear collider. For a D-T system D ions would be injected at one end, be compressed as they move up the magnetic gradient, and collide with $\mathrm{T}$ ions injected from the other end. In such a "one-pass" system the ions could be injected with energies optimized to maximize the energy yield per ion relative to the energy required to launch them. In the case of the $\mathrm{D}$ - $\mathrm{T}$ reaction these optimal energies would be $36 \mathrm{keV}$ for the $\mathrm{D}$ ions and $24 \mathrm{keV}$ for the $\mathrm{T}$ ions. If the temperature of the space-chargeneutralizing electrons is not too low, the energy loss of the ions during a single transit would be small compared to their initial energy and can be neglected to first approximation in calculating the fusion reactivity. This calculated $\sigma-v$ value is substantial higher than that of a maxwellian having the same average ion energy as illustrated in Figure 1..

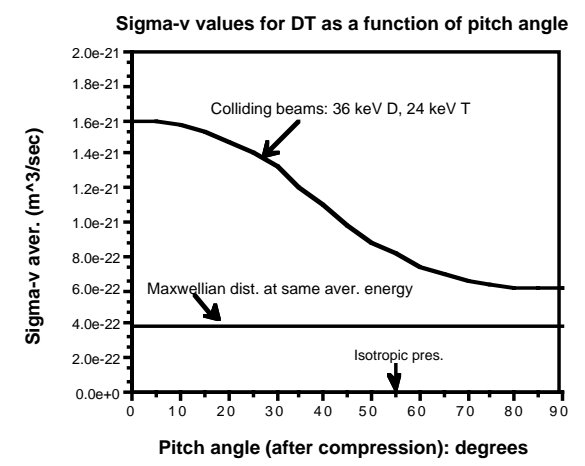

Figure 1: $\sigma-\mathrm{v}$ of colliding D-T ions

The values of $\sigma-\mathrm{v}$ shown for the case of the colliding beams are plotted as a function of the pitch angle of the ions after compression. That is, the pitch angle zero corresponds to ions injected directly along the field lines, while larger pitch angles correspond to ions injected at a small initial angle so as to arrive at the pitch angle shown.

Since the reactivity of the plasma column per unit length depends on the unrolled path length of the ions moving down the column this effect works in the opposite direction to the pitch-angle dependence shown in Figure 1. Figure 2 illustrates this effect by plotting the relative reactivity per unit length of the column as a function of pitch angle after injection.

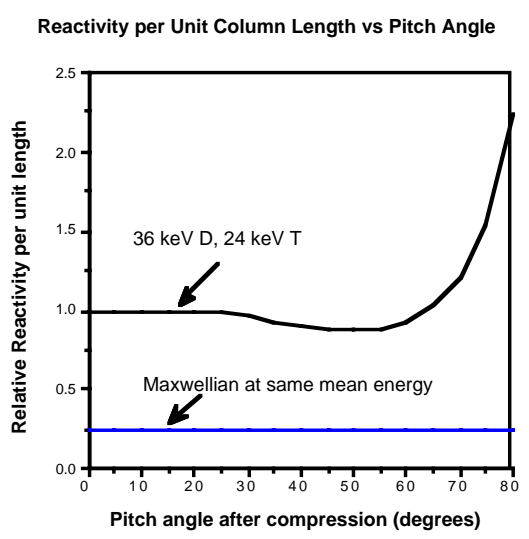

Figure 2: Fusion reactivity vs. pitch angle

In a linear collider such as we are describing the particle density of the colliding ion beams is limited by the injection current density and the ratio of the magnetic field in the central region compared to that at the point of injection. The injection current density is in turn limited by the requirement for stability against the firehose instability [19] at the point of injection. A simple calculation shows that if this stability criterion is satisfied at the point of injection it will be increasingly well satisfied at all higher values of the magnetic field. In a previous paper stability criteria relating the parameters of injection current density, ion energy, and final magnetic field to the attainable colliding-beam ion densities were presented and will be repeated here. In these criteria the ion injector current densities for the $\mathrm{D}$ and $\mathrm{T}$ ions, $\alpha_{\mathrm{D}}$ and $\alpha_{\mathrm{T}}$, are measured in units of 3 amperes/sq. cm. Although it represents an extrapolation from most present practice, this level of current density was demonstrated in early work by Dembriski and Jones [20]. The final magnetic field, $\eta$, is measured in units of 10 Tesla. The ion energies, $\lambda$, are in units of $36 \mathrm{keV}$ for $\mathrm{D}$ and $24 \mathrm{keV}$ for $\mathrm{T}$. The colliding ion densities, $\gamma_{\mathrm{D}}$ and $\gamma_{\mathrm{T}}$, are measured in units of $10^{20}$ ions $/ \mathrm{m}^{3}$. With these definitions the criteria for stability against the firehose instability are given by the expressions:

$$
\begin{aligned}
& \text { D: } 7.3\left[\frac{\lambda^{3 / 2} \gamma_{\mathrm{D}}^{2}}{\alpha_{\mathrm{D}} \eta^{2} \cos (\theta)}\right]<1.0, \text { stable } \\
& \mathrm{T}: 3.3\left[\frac{\lambda^{3 / 2} \gamma_{\mathrm{T}}^{2}}{\alpha_{\mathrm{T}} \eta^{2} \cos (\theta)}\right]<1.0, \text { stable }
\end{aligned}
$$

Upon imposing these constraints on the ion densities one can calculate the fusion power density as limited by 
the firehose instability, finding the relationship (power density in units of Watts $/ \mathrm{m}^{3}$ ):

$$
\mathrm{p}_{\mathrm{DT}} \leq 6.3 \times 10^{27}\left[\left(\alpha_{\mathrm{D}} \alpha_{\mathrm{T}}\right)^{1 / 2} \eta^{2}\right]\left\{\frac{\langle\sigma \mathrm{V}>(\lambda, \theta)}{\lambda^{3 / 2} \cos ^{2}(\theta)}\right\}
$$

\section{Example:}

$$
\begin{aligned}
& \alpha_{D}=\alpha_{T}=1.0, \eta=1.0, \lambda=1.0, \\
& \theta=75^{\circ},\langle\sigma v\rangle=6.4 \times 10^{-22} \mathrm{~m}^{3} \mathrm{sec}^{-1}
\end{aligned}
$$

With these parameters equation (3) yields a fusion power density of $60 \mathrm{MW} / \mathrm{m}^{3}$, a substantially higher power density than that for a maxwellian ion distribution at the same ion density and mean ion energy.

As described in ref. [3], this power density may be inserted in an expression for the net power input and multiplied by an energy recovery efficiency to obtain an expression for the generated electrical power, $\mathrm{p}_{\mathrm{DT}}$. This power is to be compared to the electrical power required by the beams, to determine the length of the collider required to yield net electrical power. Using the same parameters as in the example and assuming recovery efficiency values $\eta_{\text {fusion }}=0.33$ for the recovery of the fusion energy, $\eta_{\mathrm{dc}}=\eta_{\text {inj }}=0.9$ for the direct converter and injection efficiencies, respectively, one obtains for the ratio of generated power to net power required for the beams the expression:

$$
\frac{\mathrm{p}_{\mathrm{DT}}}{\mathrm{p}_{\text {beam }}}=1.4 \times 10^{-4} \mathrm{~L}_{0}
$$

Here $\mathrm{L}_{0}(\mathrm{~m}$.$) is the length of the high-field region$ of the linear collider. Thus for a collider with a length of 30 kilometers (about the same as the length of the LEPC collider at CERN), this ratio is equal to 4.2. In this example the D ion transit time is about $75 \mathrm{~ms}$. and the $\mathrm{T}$ ion transit time is about $92 \mathrm{~ms}$. so that the issue of plasma confinement is minimized.

In the absence of a detailed study one cannot have complete confidence that plasma turbulence will not play a significant role in such a system. However, there is reason to be optimistic: Ion-ion two-stream instabilities are predicted to be suppressed by the electron population, and MHD modes of either the firehose, the mirror, or the interchange variety should not be a problem. There remains the possibility of the presence of drift modes, but these would have to create transverse drifts approaching the Bohm rate to seriously interfere with the operation of the collider.
To conclude the discussion of this example, we have attempted to show that it is within the bounds of reason to consider long linear fusion colliders employing the mirror effect to enhance the density of the colliding ions. Such systems would represent a fusion approach that would place minimal requirements on confinement time, while shifting the research emphasis toward specific technological goals. In the next example we will discuss an open-ended system that might be more attractive in terms of its requirements, both as to length and as to technological demands. It should be borne in mind that neither example is offered as more than a starting point for what might turn out as an interesting area of investigation, out of which might in time come a viable approach to fusion power.

\section{B. A "Kinetic Tandem" Open-Ended System}

The second example to be discussed is what we have called a "kinetic tandem" system. The kinetic tandem idea has been presented in previous papers $[3,21]$. It represents a method of creating a confinement system that mimics that of a conventional tandem mirror, but in a magnetic field of the same type as that of the long linear collider, that is, an axially symmetric solenoidal field that is weakened at each end.

The tandem mirror concept involves "plugging" the end leakage of an open-ended field by creating localized regions of positive potential at each end of the system. In the original version of the tandem mirror $[14,15]$ the plugging potentials were created by taking advantage of the ambipolar potential that arises spontaneously in open-ended systems in equalizing the rate of loss of ions and electrons. From the quasineutrality constraint in the plasma there arises the familiar Boltzmann-like expression for the potential difference, along the magnetic field lines, between two regions of the plasma at differing densities:

$$
[e \phi(2)-e \phi(1)]=k T_{e} \ln \left[\frac{n_{e}(2)}{n_{e}(1)}\right]
$$

Thus in the original tandem-mirror concept small mirror cells, one at each end of the central mirror cell, were maintained at a higher plasma density than the plasma in the central cell, thereby creating a positive potential barrier inhibiting the escape of ions from the central cell. If the length of these cells was sufficiently short compared to that of the central cell the power required to maintain the plasmas in the plugs would be small compared to the fusion power generated in the central cell. 
In the kinetic tandem concept there are no end mirror cells within which the higher-density plasma is maintained. Instead, a region of localized higherdensity plasma near each end is to be created by the same magnetic compression process employed in the linear collider just described. Ion beams are to be injected, near the ends of the long solenoid, nearly parallel to the direction of the field lines. Moving up the magnetic gradient these ions are compressed inward and eventually slowed to a stop and reflected by the field as it approaches its maximum value in the central, "confinement cell," region. Given a sufficiently high electron temperature of the lower-density plasma in the central region, a plugging potential adequate to contain the ions of that plasma will arise, inhibiting their escape. Again, if the plasma column between the plugging potentials is sufficiently long the fusion power released in the central region will be large enough compared to the power required to maintain the plugging potentials to yield a net useful power output.

The plugs of any tandem mirror system must perform two functions: The first is to provide a potential barrier that inhibits the escape of the ions of the central plasma. The second, and equally important, function is to balance the outward-directed parallel pressure of that plasma by an equal ingoing momentum flux. The mechanism for coupling this force is the positive gradient of the electric potential acting on the ions as they enter the plugs. In the conventional tandem this momentum flux is taken up by the $\mu \nabla \mathrm{B}$ (product of magnetic moment and gradient $B$ ) force on the plug ions as they are reflected by the end mirrors of the plug cells. In the kinetic tandem this pressure-balancing requirement must be satisfied by the inward momentum flux associated with the injected and reflected ions.

The two constraints just described - sufficiently high ion density to build the plug region, plus a sufficient inward momentum flux to balance the parallel pressure of the confined plasma - must be satisfied within the additional constraints of maintaining the stability of the plug ions against two MHD modes. The first of these is the firehose mode, previously discussed, where the limitation on ion density occurs near the point of injection. The second MHD mode that must be considered is the so-called "mirror" mode [19], where the region of concern is the density maximum of the mirror-reflected plug ions.

An approximate evaluation of the first two constraints - parallel pressure and plug ion density - may be obtained from simple mirror-physics relations. Ions of mass $\mathrm{M}_{\mathrm{i}}(\mathrm{kg})$, injected at a current density $\mathrm{j}_{0}$ (ions $/ \mathrm{m}^{2}$-sec.) at a velocity of $\mathrm{v}_{0}(\mathrm{~m} / \mathrm{sec}$.) and at an angle to the direction of the magnetic field of $\theta_{0}$ (radians) produce an inward momentum flux that is, at first, increased by the convergence of the field lines, and then decreased as their perpendicular component of energy is increased through mirror action. As a result the parallel pressure reaches its maximum value at a field ratio that is $2 / 3$ of that where reflection occurs, i.e. at a field ratio given by the relationship:

$$
\frac{\mathrm{B}(\mathrm{z})}{\mathrm{B}(0)}=\frac{2}{3 \sin ^{2}\left(\theta_{0}\right)}
$$

At this point the parallel pressure has the magnitude:

$$
\mathrm{p}_{\mathrm{par}}(\max )=\frac{2}{3 \sqrt{3}} \mathrm{j}_{0} \quad \mathrm{M}_{\mathrm{i}} \mathrm{v}_{0}\left[\frac{1}{\sin ^{2}\left(\theta_{0}\right)}\right]
$$

At the maximum point the ion density from these ions has been compressed up to the value:

$$
\mathrm{n}_{\mathrm{i}}=\frac{2 \mathrm{j}_{0}}{\mathrm{v}_{0}}\left[\frac{1}{\sin ^{2}\left(\theta_{0}\right)}\right] \quad \text { ions } / \mathrm{m}^{3}
$$

For sufficiently small values of the injection angle and for ion source current densities of the order of those previously cited both the parallel pressure and the compressed ion density reach levels of practical interest. In what follows we will present more detailed evaluations of these quantities.

1. Calculation of plug parameters. Prior to actual experimentation, the best way to evaluate the plasma properties and the parameters of the plugs of a tandem mirror would be through the use of plasma simulation codes. In the absence of such simulations theory-based calculations can provide some insight. We will present the results of such calculations in the material to follow.

To obtain a lowest-order answer to the density compression and the parallel pressure increase caused by the injection of a beam of ions into a rising magnetic field the classical technique of employing a distribution function written in terms of the invariants of motion, namely, the specific energy, $\varepsilon=\mathrm{v}^{2} / 2$, and the specific magnetic moment, $v=v_{\perp}^{2} / 2$. The angular dependence of the ion source function, $g(\theta)$ was chosen to representative of realistic ion sources, while at the 
same time being integrable in terms of simple functions. The form chosen was:

$$
g(\theta)=g_{0}\left[\sin ^{2}(\theta)-\sin ^{2}\left(\theta_{1}\right)\right]^{2}\left[\sin ^{2}\left(\theta_{2}\right)-\sin ^{2}(\theta)\right]^{2}
$$

A plot of this distribution function for typical values of the injection angle and angular width is shown in Figure 3.

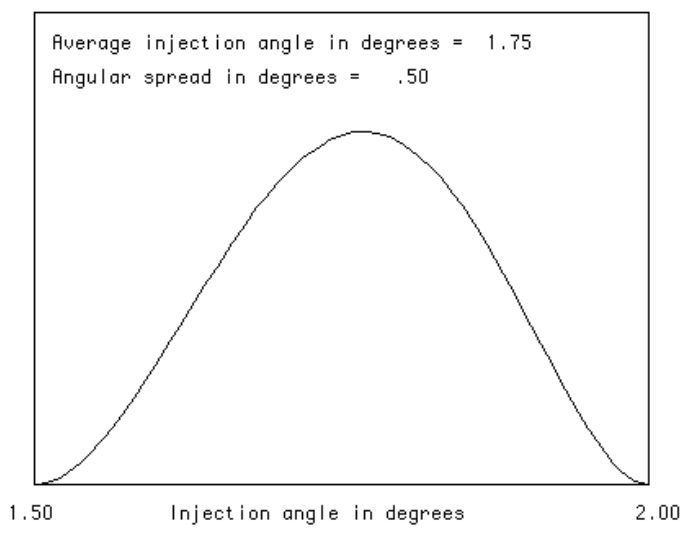

Figure 3: Source Angular Distribution

Inserting this distribution function into the expression for the density, integrating, and normalizing to the density at the position of the source resulted in plots of this density ratio as a function of the magnetic field intensity. One such plot is shown in Figure 4. In the plot the ion density is shown to be compressed by a factor of 6085 , with the peak density occurring at a field ratio (ratio of magnetic field at the density peak to that at the ion source) of 960:1.

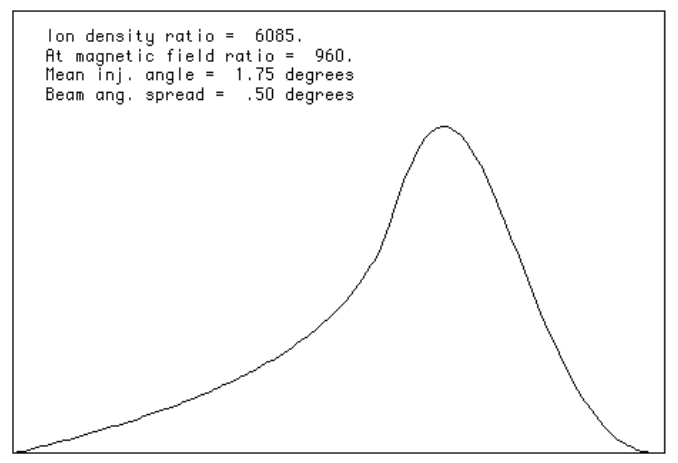

Figure 4: Density compression vs. magnetic field

The same analytical approach can be used to obtain the perpendicular and parallel pressure amplifications. These are shown on Figures 5 and 6, respectively.

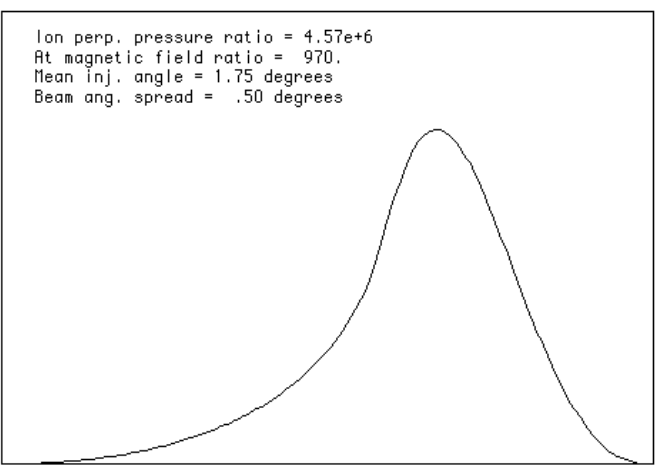

Figure 5: Perpendicular pressure vs. magnetic field

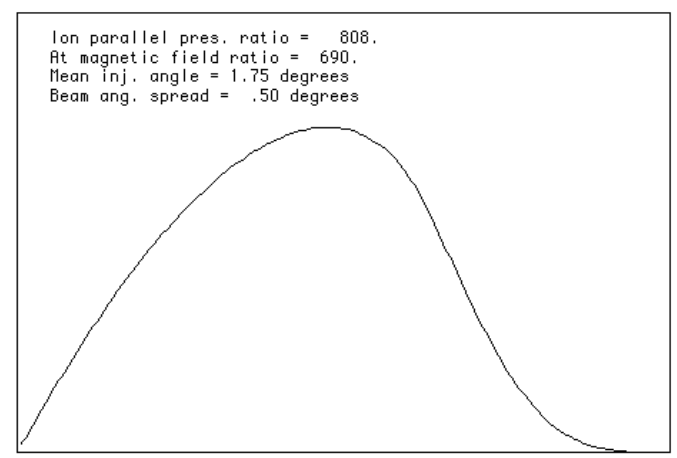

Figure 6 Parallel pressure ratio vs. magnetic field

2. Calculation of stability requirements. Knowing the perpendicular and parallel pressure ratios and their absolute value permits the calculation of a stability parameter for the mirror instability. Written in terms of the pressure and magnetic field parameters we define a quantity $\mathrm{H}$ :

$$
H=\left\{\left[\frac{p_{\text {perp }} \text { (ion.) }}{p_{\text {par }}}\right] \cdot\left[\frac{p(\text { perp })}{p(\text { par })}-2\right] \cdot\left[\frac{B_{\text {max }}^{2}}{B_{z}^{2}}\right]\right\}
$$

Having evaluated the quantity $\mathrm{H}$ from the calculated pressure values as a function of magnetic field one then compares this to the stability criterion, stated in terms of field and pressure quantities:

$$
\mathrm{H}<\left[\frac{\mathrm{B}_{\max }^{2}}{\mu_{0} \mathrm{p}_{\mathrm{par}}(\max )}\right] \text {, stable }
$$

In terms of the parameters used before this criterion becomes:

$$
\mathrm{H}<\frac{10^{2} \eta^{2}}{\mu_{0} \mathrm{p}_{\mathrm{par}}(\max )} \text {, stable }
$$


If we now simultaneously impose the firehose instability criterion the final criterion for stability against both modes is the following:

$$
\mathrm{H}<\frac{4.03 \times 10^{2} \lambda_{\text {inj }}^{1 / 2} \gamma_{\mathrm{e}}(\max )}{\mathrm{ZA}^{1 / 2} \mathrm{~F}} \text {, stable }
$$

The new parameters here are $\lambda_{\text {inj }}$, the plug ion beam energy in units of $10 \mathrm{keV}, \gamma_{\mathrm{e}}(\max )$, the electron density at the maximum perpendicular pressure and $\mathrm{F}$, which is the ratio of the electron density at the point of maximum density to that at the point of maximum parallel pressure. From the plots we find that for the cases shown $F=4.0$ to a close approximation.

Example:

$$
\lambda_{\text {inj }}=1.0, \gamma_{\mathrm{e}}(\max )=10.0 \text { (elec. density }=10^{21} \mathrm{~m}^{-3}
$$
) ,

$$
\mathrm{Z}=3, \mathrm{~A}=7 \text { (lithium), } \mathrm{F}=4.0
$$

We then find for the overall stability criterion the requirement:

$$
\mathrm{H}<126 \text {, stable }
$$

All of the required functions can be programmed in from results such as those presented in Figures 4, 5, and 6 in order to produce a plot of the function $\mathrm{H}$ as a function of magnetic field. The maximum point on this point, corresponding to the most nearly unstable region in the plug plasma, can then be compared to the stability criterion to see if it is satisfied. Figure 7 represents such a plot, made using the same beam parameters as those used in the plots and the example.

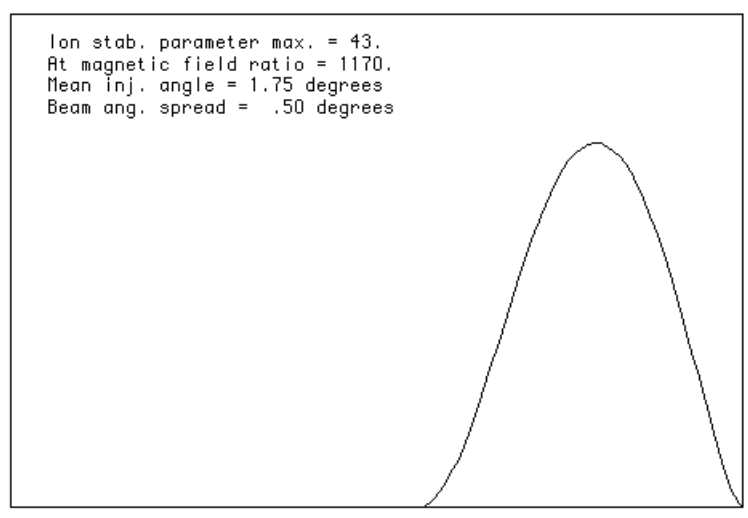

Figure 7: Plot of the stability parameter $\mathrm{H}$ vs. $\mathrm{B}$
From the plot it can be seen that the maximum value of $\mathrm{H}$ over the entire range of $\mathrm{B}$ is 43 , well below the stability boundary, $\mathrm{H}<126$, calculated in the example. Since the numbers that were used in the example represent fusion-relevant values, it seems probable that stability against both the firehose and the mirror mode can be achieved in the plugs of the kinetic tandem at practical values of magnetic field and fusionrelevant particle densities. It is worth noting that some experiments have already been performed that are supportive of this conclusion. For example, the measurement of "sloshing ion" populations in mirror cells [22] that were performed by the Novosibirsk mirror research group show no evidence of instabilities interfering with the peaking of ions at their point of mirror reflection.

\section{Modes of Operation of the Kinetic Tandem}

Having established a means for generating localized regions of positive potential using the kinetic tandem concept there are various ways that the idea might be employed in conceptualizing a fusion power system. As an example we will consider a "two-ion-component" type of system. In a two-ion-component fusion power system a beam of energetic fuel ions, for example $\mathrm{T}$ or He3, collide with other fuel ions, for example D ions in a higher density "target" plasma of modest kinetic temperature. The fusion power released is proportional to the product of the two ion densities and to the integrated reactivity of the energetic ions as they slow down in moving through the target plasma.

In the early 1970 s studies were carried out on twoion-component tokamak fusion power systems [23]. Following these studies similar calculations were made for mirror-based systems [24]. For our discussion we will consider long linear systems whose central region contains a target plasma at modest kinetic temperature (a few kilovolts). Into this plasma will be injected ions whose energy is many tens of kilovolts, high enough to approach the maximum $\sigma-\mathrm{v}$ value for the fuel ions involved.

The simplest form of two-ion-component kinetic tandem fusion power system is one in which the energetic ions are injected at one end and make a single pass through the target plasma. Such a system would make minimal demands on the required confinement time of the energetic ions, but would place maximal demands on the efficiency of the ion injectors and of 
the direct converters, and would require a long system to yield net power.

In considering the power-balance requirements for kinetic tandem mirror systems three different loss channels must be considered. The first of these is the power required to produce and maintain the potential peaks. The second one is the power loss associated with the target plasma, determined by its mean energy containment time. The third loss channel is associated with the injection and energy recovery from the energetic fuel ions. Each of these must be compared with the fusion power output in establishing the overall fusion power balance.

Considering the first loss channel, the power required to maintain the potential peaks, an expression has been derived that can be used to estimate the minimum column length of fusing plasma that would be required to supply the power needed to maintain the plug. The expression is (power in Watts $/ \mathrm{m}^{2}$ ):

$$
P_{\text {loss }}=1.36 \times 10^{11}\left[\frac{\lambda_{\text {inj }}^{3 / 2} \gamma_{\mathrm{e}}(\max )}{\mathrm{ZFA}^{1 / 2}}\right]\left[1 / \eta_{\text {inj }}-\eta_{\mathrm{DC}}\right]
$$

\section{Example:}

0.9

$$
\begin{aligned}
& Z=3, A=7 \text { (Lithium), } F=4.0, \gamma_{\mathrm{e}}(\max )=1.0 \\
& \left(\mathrm{n}_{\mathrm{e}}=10^{20} \quad \mathrm{~m}^{-3} \text { at peak }\right), \lambda_{\text {inj }}=1.0, \eta_{\mathrm{inj}}=\eta_{\mathrm{DC}}=
\end{aligned}
$$

$$
\mathrm{P}_{\text {loss }}=9.0 \times 10^{8} \mathrm{Watts} / \mathrm{m}^{2}
$$

At, for example, an assumed fusion power density of $30 \mathrm{MW} / \mathrm{m}^{3}$, recovered at an efficiency of 0.33 , equation (15) predicts the need for a plasma column about 90 meters in length to generate the electrical power needed to sustain each plugging potential peak. If it is found to be technically feasible to employ plug ions with a higher $\mathrm{Z}$ and $\mathrm{A}$ value than the example case chosen (Lithium) then the plug power requirements could be lowered accordingly. Clearly, however, the kinetic tandem idea is best suited for use in long linear systems.

Considering the second loss channel, the target plasma, rough estimates indicate that its losses would be acceptably low, so long as the radial transport losses are reasonably small compared to the Bohm-diffusion rate.

Following up on the estimate given by equation (15), we have made a calculation of the ratio of the fusion power generated to the power required to maintain the potential peaks for a two-component linear system and find it satisfactory for the long linear systems we are considering. The calculations were made for the case of energetic $\mathrm{T}$ ions injected into a target plasma composed of $\mathrm{D}$ ions. The expression derived can be written in terms of two factors: The first factor contains the beam and plug ion parameters previously defined. The second factor contains the injection and energy recovery efficiencies and the system length. The form of the expression is:

$$
\begin{aligned}
& \frac{\mathrm{P}_{\text {fusion }}}{\mathrm{P}_{\text {plug }}}=\mathrm{K}_{1}(\eta, \mathrm{F}, \ldots) \cdot \mathrm{K}_{2}\left(\mathrm{~L}_{0}, \ldots\right) \\
& \mathrm{K}_{1}=2.0 \times 10^{-4}\left[\frac{\mathrm{G} \alpha_{\mathrm{T}}^{1 / 2} \eta \mathrm{ZA}^{1 / 2} \mathrm{~F}}{\lambda_{\mathrm{i}}^{3 / 2} \cos ^{2}(\theta)}\right] \\
& \mathrm{K}_{2}=\left[\frac{\eta_{\text {fus }}}{\left(1 / \eta_{\text {inj }}\right)-\eta_{\mathrm{dc}}}\right] \mathrm{L}_{0}
\end{aligned}
$$

\section{Example:}

$$
\eta_{\text {inj }}=\eta_{\text {dc }}=0.8, \eta_{\text {fus }}=0.33, \mathrm{G}=0.5 \text { (ratio of }
$$

electron density in target plasma to electron density at potential peak of plugs $), \eta=1.0(10$ Tesla central field), $\mathrm{Z}=3, \mathrm{~A}=7$ (lithium plug ions), $\mathrm{F}=4.0, \cos (\theta)$ $=0.5, \lambda_{\mathrm{i}}=1.0(10 \mathrm{keV}$ plug ion energy $)$.

$$
\text { At } \mathrm{L}_{0}=3 \times 10^{4} \text { meters, } \quad \frac{\mathrm{P}_{\text {fusion }}}{\mathrm{P}_{\text {plug }}}=70
$$

As can be seen the estimated power ratio factor is sufficiently robust to allow substantial changes in the parameters without seriously compromising the fusion power balance. The example given here is simply to show that in a sufficiently long linear system the power to maintain the plugs should represent a reasonably small fraction of the fusion power output. This is the same assumption that is made in considering conventional tandem mirror systems.

\section{Kinetic Tandem Plugs: Further Considerations}

The calculations and estimates that have thus far been given on the kinetic tandem concept have been based on zeroth-order analysis. That is, the spatial distribution of the ion density in the plugging regions has been calculated ignoring the effect of the potentials thus produced on the motion of the ions. Such selfconsistent-field problems are best handled by PIC computer codes or their equivalent. In the absence of 
such code calculations we have attempted to perform next-order calculations using the following method:

The analytical problem was rephrased by introducing a to-be-determined plasma potential (a function of the local value of $B$ ) into the integral expression for the ion density in the plugs. This potential in turn would be calculated using the Boltzmann assumption (equation 5). By a transformation in variables the density equation was put in a form suitable for numerical integration. Using this formulation the calculation of the density ratios was first performed with the electron temperature set to zero (i.e. zero potential at all $B$ values). The results of this calculation were found to agree closely with the analytical results presented earlier (Figure 4). By an iterative procedure the electron temperature was then gradually increased and the potential and its spatial distribution were then calculated, inserting the resulting density distribution in equation 5 at each step to evaluate the potential. At each step the potential distribution in the new calculated self-consistent density distribution was then used to calculate the next density distribution, and so forth. The results of these calculations, for the same ion angular distribution as that in Figure 4, is shown in Figure 8. In the figure two plots are shown. The right-most plot is for the case of zero electron temperature, i.e. it reproduces the results of Figure 4. The plot whose peak lies to the left of that plot is the one obtained by the iterative procedure for a case where the ratio of the electron temperature to the ion plug energy is 0.025 . That plot is shown only up to its maximum point as the iterative procedure that was used is valid only over that range of B values where the potential remains single-valued.

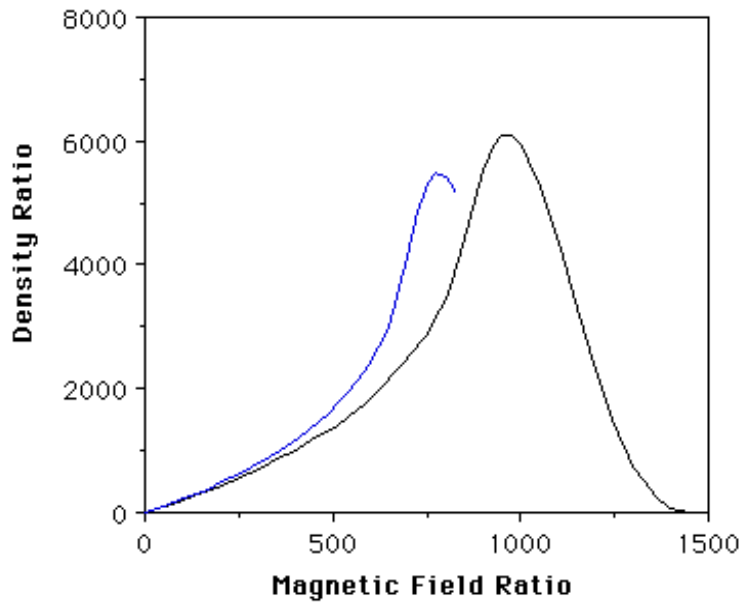

Figure 8: Density ratio: effect of potential
As is evident from Figure 8, the effect of the potential is to cause the injected ions to be reflected at a lower field value than when the potential is zero, forming a "virtual anode." Possibly advantage could be taken of this effect to build the potential to higher values. For example, once the potential begins to build, ions of somewhat lower energy could be injected so as to "pile up" near the peak, thereby enhancing the local ion density, and with it the potential. Such possibilities, including the possibility of unstable behavior associated with these effects, could be investigated with computer simulations or experiments.

Other methods of enhancing the potential in the kinetic tandem include the use of localized heating of electrons near the potential peak. In the presence of the magnetic gradient these electrons would feel an outward force. Owing to quasi-neutrality the expected response of the system would be to increase the potential.

If it turns out to be practical, the concept involved in the kinetic tandem, namely, the production of localized regions of positive potential in an open-ended solenoidal field by the injection of directed ion beams, might turn out to be of value in other contexts. The fact that the potential can be controlled by modulation of the injected ions could be useful in ash removal or in the trapping of injected fuel ions. It also could be useful, at lower potential values, for the confinement of secondary plasmas, e.g. for "warm plasma stabilization," or for plasma employed in the ionization of transiting neutral beams

\section{TECHNOLOGY ISSUES}

A stated purposes of this paper is to encourage a shift in emphasis of magnetic fusion research. The shift: away from systems where the main issue is increasing plasma confinement to the end of achieving plasma ignition, and toward low-Q open-ended systems where the field geometry promotes plasma stability, where the confinement requirements are relaxed and the physics involved is better understood. The effect would be to enhance the importance of some key technologies, the pursuit of which is less likely to be fraught with the frustrations (complexity and theoretical intractability, etc.) that have been met in pursuing the tokamak approach.

The key technologies, already alluded to, include efficient means for the injection of ion (or neutral atom) beams at high current densities, with narrow angular distributions, and with high electrical efficiency. 
Another would be that of the electrostatic direct converter. Although good work was done in this area decades ago new issues would arise in connection with the new approaches. For the kinetic tandem, among these issues would arranging for geometric compatibility of the ion injection system and the direct converters. The value of these developments is that every improvement would enhance the performance of the system, either making it more efficient, smaller, or less expensive. Finally, as mentioned earlier, the enhancement of these key technologies would work hand-in-hand with a push toward fusion power systems based on alternative fusion fuels, such as the D-He3, the p-Li6, or the p-B11 cycles.

\section{SUMMARY AND CONCLUSIONS}

The pursuit of magnetic fusion research has had different goals over the nearly 50 years that it has been conducted. In the earliest days "the first thermonuclear neutrons" was a chimerical goal that was only a diversion. This goal was replaced by a more reasonable, but still stultifying one, the pursuit of ever higher containment $n \tau$ values. A positive effect of pursuing this goal was to focus on plasma instabilities and ways to ameliorate their effect on confinement. A negative effect was to cause, worldwide, the near abandonment of approaches that did not obviously extrapolate to high confinement $n \tau$ values, e.g. the mirror machine. In later years the goal has shifted toward the achievement of ignition of a D-T plasma, even if it has to be achieved in systems whose long-range value is questionable. We are now in the process of rethinking these goals. This paper has attempted to point out some potentially promising areas to be considered and to suggest some avenues that appear to be open to innovative improvements. The examples presented may not stand the test of time. However, the impetus behind presenting them is to encourage thinking in new ways about the fusion problem and its solution. Fusion is still humankind's best hope for its energy future!

\section{ACKNOWLEDGMENTS}

In the preparation of this paper the author benefited from valuable discussions with D. D. Ryutov and L. D. Pearlstein. The work was carried out under the auspices of the U. S. Department of Energy by the Lawrence Livermore National Laboratory under contract no. W-7405-Eng-48.

\section{REFERENCES}

1. R. F. Post, "Comments on Open-Ended Confinement Systems for Fusion," Physics of Alternative Magnetic
Confinement Schemes, p 63, Societa Italiana diFisica (1981)

2. R. F. Post, J. F. Santarius, “ Open Confinement Systems and

the D-He3 Reaction ," Fusion Technology, 22, 13 (1992)

3. R. F. Post, "Open Systems in the Quest for Fusion," Plasma Physics Reports, 23, 816 (1997)

4. L. Roberts, Kiewet Construction Co., (private communication).

5. C. Luongo, "Superconducting Storage Systems: An Overview," IEEE Trans. on Magnetics,32, 2214 (1996)

6. R. F. Post, D.D. Ryutov, "Open-Ended Confinement Systems for Fusion," Current Trends in International Fusion Research, p. 153, Plenum Press, New York, New York (1997)

7. D. L Correll, J. F. Clauser, F. H. Coensgen, "Production of Large-Radius, High-Beta, Confined Mirror Plasmas," Nuclear Fusion, 20, 655 (1980)

8. J. B. Taylor, R. J. Hastie, "Maximum Plasma Pressure for Stability in Magnetic Fields with Finite Minima," Physics Fluids, 8, 323 (1965)

9. F. H. Coensgen, W. F. Cummins, W. E. Nexsen, Jr., A. E. Sherman, "Production and Containment of Hot Deuterium Plasmas in Multistage Magnetic Compression Experiments," Nuclear Fusion 1962 Supplement, p. 125 (1962)

10. R. F. Post, "Mirror Systems: Fuel Cycles, Loss Reduction and Energy Recovery," Nuclear Fusion Reactors, British Nuclear Eng. Soc., p. 88 (1969)

11. R. W. Moir, W. L. Barr, G. A. Carlson, Plasma Physics and Controlled Nuclear Fusion Research, 1974, Vol. 3, p. 583, IAEA Vienna (1975)

12. F. H. Coensgen, O. A. Anderson, T. A. Casper, et.al., "Electrostatic Plasma Confinement Experiments in a Tandem Mirror System," Phys. Rev. Lett. 44, 1132 (1980)

13. T. Cho, M. Ichimura, M. Inutake, et. al., Plasma Physics and Controlled Nuclear Physics Research 1984, Vol. 2, p. 275, IAEA Vienna (1985)

14. G. I.. Dimov, V. V. Zakaidov, M. E. Kishinvevskii, 
"Thermonuclear Confinement System with Twin Mirror Systems," Sov. J. Plasma Phys., 2, 326 (1976)

15. T. K. Fowler, B. G. Logan, "The Tandem Mirror Reactor," Comments Plasma Phys. Controll. Fusion, 2, 167 (1977)

16. T. C. Simonen, S. L. Allen, D. E. Baldwin, et. al., Plasma Physics and Controlled Nuclear Physics Research 1986, Vol. 2, p. 231, IAEA Vienna (1987)

17. M. Inutake, A. Ishimura, R. Katsumata, et. al., International Conference on Open Plasma Confinement Systems for Fusion, Editor A. A. Kabantsev, p. 51, World Scientific (1994)

18. D. D. Ryutov, G. V. Stupakov, "Transverse Particle Losses in an Ambipolar Trap,” JETP Lett. 26, 174 (1977)

19. W. A. Newcomb, "Equilibrium and Stability of Collisionless Systems in the Paraxial Limit," J. Plasma Phys., 26, 529 (1981)

20. M. Dembinski, P. K. John, "Efficient Ion Extraction from a Flowing Plasma,” J. Appl. Phys., 50, 6113 (1979)

21. R. F. Post, "Open-Ended Systems: Some Possible New Directions," IAEA Tech. Committee Meeting: "Innovative Approaches to Fusion Energy," LLNL Report CONF 971079 (1997)

22. P.A. Bagryanskii, A. A. Ivanov, et. al., Plasma Physics and Controlled Nuclear Fusion Research 1990, Vol. 2, p. 655, IAEA Vienna (1991)

23. J. M. Dawson, H. P. Furth, F. W.. Tenney, "Production of Thermonuclear Power by Non-Maxwellian Ions in a Closed Magnetic Field Configuration," Phys. Rev. Lett., 26, 1156 (1971)

24. R. F. Post, T. K. Fowler, J. Killeen, A. A. Mirin, "Concept for a High-Power-Density Mirror Fusion Reactor," Phys. Rev. Lett., 31, 280 (1973) 


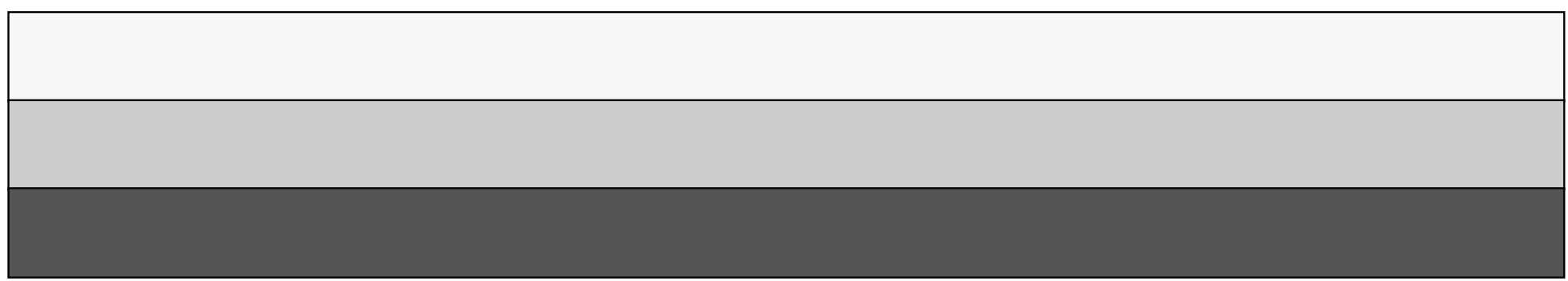

